\title{
Analysis of the process of green hemp stalks sequential harvesting
}

\author{
Anişoara Păun ${ }^{1 *}$, Gheorghe Stroescu ${ }^{1}$, Alexandru Zaica ${ }^{1}$, Radu Ciupercă $^{1}$ and Constantin \\ Bogdanof $^{1}$ \\ ${ }^{1}$ INMA Bucharest, Romania
}

\begin{abstract}
HEMP (Cannabis Sativa - Industrial Hemp) is the industrial plant with the highest use of all these plants in the industry, since nothing is thrown away from this plant. Hemp is the plant of which everything is used, and the products obtained are of a great diversity, from the common rope to the medicinal or cosmetic substances, textiles, automotive or construction materials. It is an unpretentious plant because, apart from the fact that it wants a neutral $\mathrm{PH}$ and soils without excess humidity, no maintenance work need to be done and no extra care should be given. The fibre hemp is harvested at the technical maturity when the male plants shed the last pollen grains, and the stalks have a greenish-yellow colour and the leaves fell on the ground. Production management is different for an exclusive fibre production. If we look for raw materials that meet the demands of different areas of the economy with the lowest costs, we must take into account the reactivation of the hemp culture at the level of Romania, because, as we mentioned above, hemp is the plant of which everything is capitalised, and the products obtained are of a great diversity. By applying the technology for processing the stalks in green, the obtained fibre can be used with special results in the strawberry culture at the mulching operation. The paper presents the Equipment for harvesting green hemp stalks - ERCV, equipment that performs sequential harvesting of hemp stalks leaving them on the ground. At the same time, factors that influence the process of harvesting hemp stalks are presented: harvesters' cutting devices, kinematic of the cutting blades, cutting speed, cutting angles of the cutting blades, the forces acting on the blade and the power required to drive the cutting devices.
\end{abstract}

\section{Introduction}

Considering that raw materials that meet the needs of the different areas of the economy with the lowest costs are sought, the reactivation of the hemp culture in Romania must be taken into consideration as well, because from this plant everything is used and the products obtained are of a great diversity. Hemp is an unpretentious plant because, apart from the fact that it wants a neutral $\mathrm{pH}$ and soils without excess moisture, no maintenance work should be done and no extra care should be given. [1-3].

\footnotetext{
${ }^{*}$ Corresponding author: ani_paun@yahoo.com
} 
The costs per hectare are below the costs of other well-known crops such as rape or wheat. At the same time after the cultivation of a land with this plant, the following year the soil becomes favourable for the cultivation of cereals because hemp naturally contributes to the removal of weeds, creating an inappropriate environment for their development [4].

Hemp seeds are used to make beer, oil used in the paint industry, cosmetic oil, edible oil or margarine. Medicinal teas and medicines are made from hemp leaves.

Hemp seed oil is extremely valuable for Omega 3, Omega 6, Omega 9, Vitamin E, being used by consumers to help the cardiovascular system. A lot of food can be provided from hemp. Hemp seeds are an important source of nutrients in large quantities. It is known that hemp seeds, in the form of flour, oil, cold extract, consumed raw or fried, are a healthy food and a non-medicinal remedy effective in many medical conditions. Hemp fibre has excellent moisture absorption properties, bacteriostatic, heat-resistant and antistatic properties; the hemp stalk is also a raw material for obtaining paper recently widely used for composite plates and other composite materials. At the same time, by applying the technology for processing the stalks in green, the obtained fibre can be used with special results in the strawberry culture at the mulching operation [5-6].

At this time the demand for hemp on the European market is increasing, and the value of the subsidies offered by the European Community helps the farmers to make a profit. In Romania only seeds are used, the textile industry not being interested in the hemp fibre.

Before 1989, in Romania, over 50,000 hectares were cultivated with hemp, but in 2000 the hemp disappeared from the Romanian agriculture, only after 2012 the cultivation of hemp began to get the attention of Romanian farmers. Romania offers favourable conditions for hemp cultivation over most of its territory. The success of hemp for both fibre and seed is given by its location in specialized farms well organized and technically equipped, in areas with pedoclimatic conditions. Following discussions with farmers and researchers from the research centres, they want to develop machines that allow them to harvest and process the stalks in green because there have been demands on the hemp market for the fibre, but especially for the green inflorescence part. Worldwide, there are companies that make such machines but at very high prices for Romanian beneficiaries. In this sense, within our unit was developed technical harvesting equipment that takes into account the technological requirements specific to the Romanian hemp varieties, in the case of harvesting hemp for fibre: the average cutting height from the ground does not exceed 10 $\mathrm{cm}$; stalk losses are less than 5\%; broken stalks are up to 6\% [7-8].

The main objective of this paper is an analysis of the process of green hemp stalks sequential harvesting.

\section{Methodology}

The hemp for fibre is harvested at technical maturity, when the male plants shake their last pollen traces, and the stalks have a greenish-yellow colour and the leaves fallen to the ground. Production management is different for an exclusive fibre production. The selection of varieties is oriented towards the production of biomass. Harvesting hemp for fibre is done at the end of flowering, when the stalks have become golden-yellow. The main features of hemp stalks are: length (total hemp length), thickness, colour, attack of diseases and pests, mechanical injuries (hail, broken or crushed stalks), and breaking load.

The length and thickness of the stalks are influenced by many factors such as: variety, sex, day length, soil and air humidity, temperature, physical and chemical composition of the soil, applied agrotechnics, fertilizers, culture system, plant density in the field etc. In the case of hemp, the stalks with the biggest useful length, not branched and thinner, are considered to be the most valuable. They have the highest fibre content, give a high fibre yield, and the obtained fibre is more resistant. 
The experimental model of Equipment for harvesting green hemp stalks, Figure 1, is intended for sequential harvesting of hemp stalks, in order to process them to obtain the fibre.

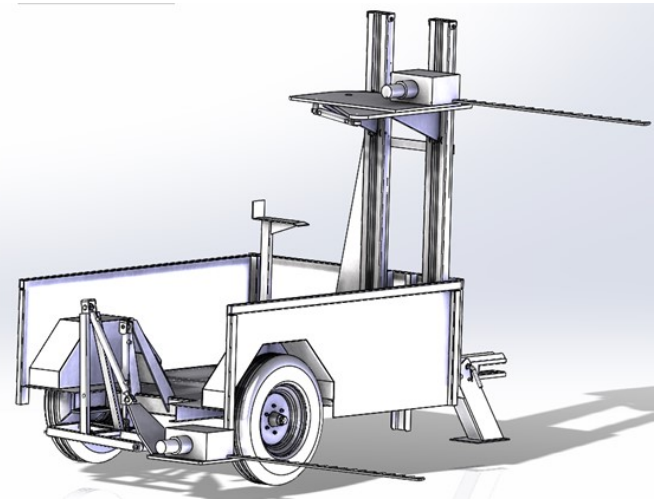

Fig. 1. Equipment for harvesting green hemp stalks ERCV: 3D

When using this equipment (description in fig 4) the cut plants remain on the ground, with the inflorescence mixed with the stalks. It should be noted that the equipment is intended for small and medium-sized farms that grow hemp.

The machine is equipped with two cutting devices, Figure 2, fixed on a mobile platform. A device is mounted on the back of the platform and cuts the stalks at a height of $100 \mathrm{~mm}$ from the ground in the working position; it is a double knife type with double cutting device, in which the role of the fingers and counter-cutting plates is fulfilled by the second knife moving at the same speed and in the opposite direction as the first knife.
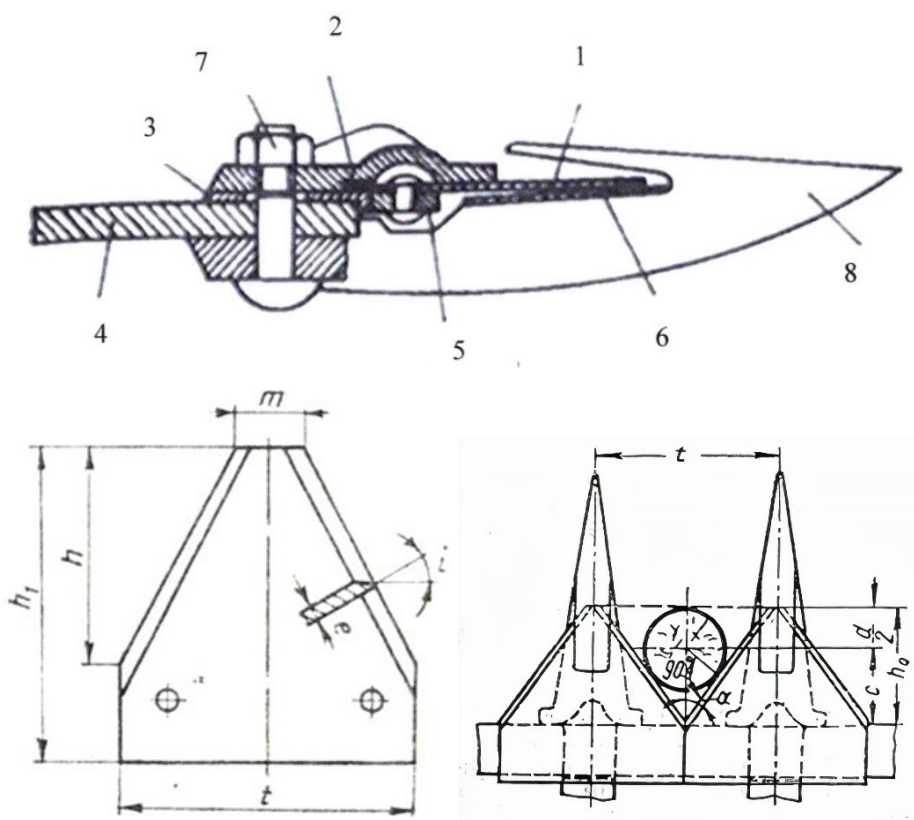

Fig. 2. Parts of the cutting device: 1 - Knife; 2 - Pressure plate; 3 - Wear plate; 4 - Cutting device support; 5 - Plate holder bar; 6 - Counter knife; 7 - Attachment screw; 8 - Peak

The simple stroke of the cutting device shown in figure 2 is determined by the relation: 


$$
S=t=t_{0}=76.2 \mathrm{~mm}
$$

Where:

$S$ - is the knife stroke at the half turn of the crank;

$t$ - the distance between the axes of two blades (step of the blades);

$t_{0}$ - the distance between the axes of two fingers (step of the fingers).

For actuating the knives of the cutting devices, axial crank-connecting rod mechanisms are used, at which $\mathrm{r} / \mathrm{l}$ has small values, the movement of a point on the knife can be considered as a harmonic oscillation. The kinematics of the knives used on the equipment for harvesting green hemp stalks ERCV is shown in Figure $3 \mathrm{a}$ and $\mathrm{b}$.
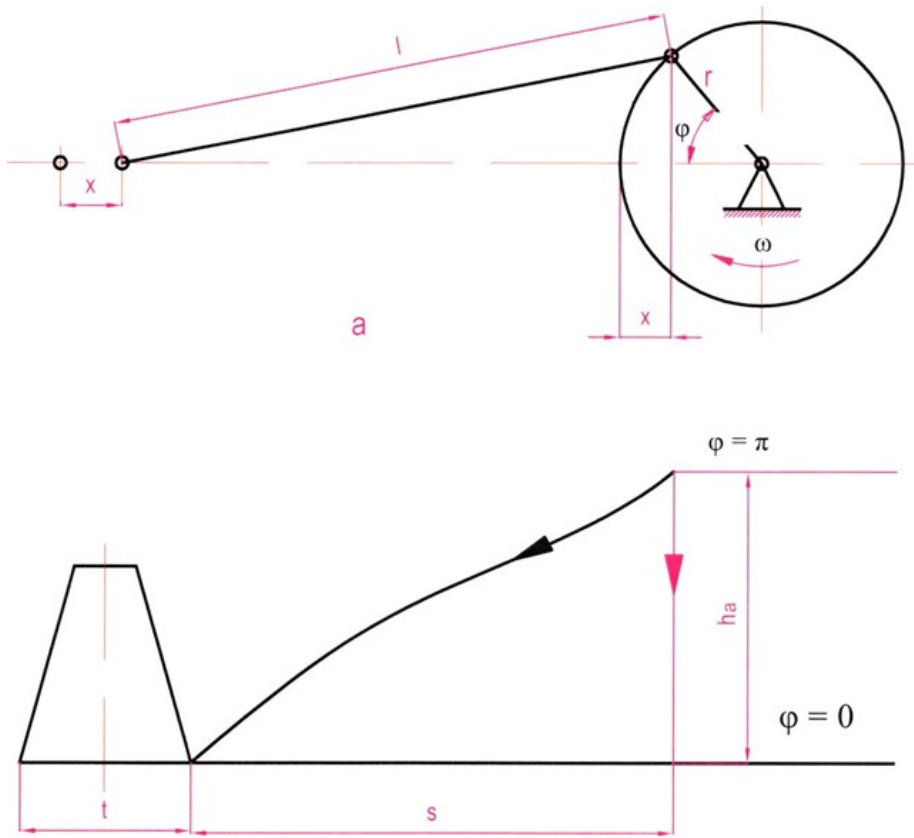

Fig. 3. The kinematics of the knife

In this case the space $x$ will be:

$$
x=r-r \cos \varphi=r(l-\cos \cdot \omega t)
$$

where:

$\omega$ - the angular speed of the crank, in $\mathrm{rad} / \mathrm{s}^{-1}$;

$t$ - the time the crank rotates with the angle $\varphi ; \varphi=\omega t$.

When the crank rotates with $\varphi=\pi$, the knife covers the space

$$
x=S=r(1-\cos \cdot \pi)=2 r
$$

where:

$S$ is the knife stroke

The speed of the knife is 


$$
v=\frac{d x}{d t}=\omega \cdot r \cdot \sin \omega t
$$

and the acceleration of the knife is:

$$
a=\frac{d v}{d t}=r \cdot \omega^{2} \cos \omega t
$$

From the expression (4) it can be noted that $v=0$ when $\varphi=0$ or $\pi$. When $\varphi=\pi / 2$, the speed of the knife is maximum and equal to $r \cdot \omega$.

The knife's acceleration has maximum values $\left(a_{\max }= \pm r \cdot \omega^{2}\right)$, when $\varphi=0$ or $\varphi=\pi$

For $\varphi=\pi / 2, a=0$.

During work, a point on the knife has two movements: of translation with the speed $v_{m}$, in the direction of machine movement and a harmonic oscillatory movement perpendicular to the direction of machine's advance. The trajectory described by a point on the knife is a sinusoid (3b). At a knife stroke $(\varphi=\pi)$ the car covers a feed space with the speed $v_{m}$ :

$$
h_{a}=v_{m} t=v_{m} \frac{\pi}{\omega}
$$

In general, the values of the feed space ha are $50-100 \mathrm{~mm}$.

Cutting the plants in good conditions depends on the cutting speed $v$, feeding $l$, the fingers' step $t_{0}$, the type of cutting edges (smooth or toothed), the sharpness of the knife edge and the distance between the cutting blade and the counter-cutting plate.

The speed of a certain point of the cutting blade, which is at a distance $x$ from the initial position, is determined by the relation:

$$
v_{x}=S \omega \sqrt{\frac{x}{S}\left(1-\frac{x}{S}\right)},[\mathrm{m} / \mathrm{s}]
$$

and the complete stroke of the knife, taking into account the offsetting $i$, is determined by the relation

$$
S=\sqrt{(u+r)^{2}-i^{2}}-\sqrt{(u-r)^{2}-i^{2}}
$$

Where : $\omega=\frac{\pi \cdot n}{30}$, is the angular speed of the crank;

$u$ - grain length

$r$ - radius of the crank

The relative average speed is calculated with the relation $v_{c}=\frac{S n}{30}$, and the maximum one with the relation $v_{\max }=\frac{S \omega}{30}$.

The absolute average speed of the knife 


$$
v_{a}=\sqrt{v_{m}^{2}-v_{c}^{2}}
$$

The acceleration of any point of the cutting blade that is at a distance $x$ from the initial position is determined by the relation

$$
a_{x}=\omega^{2}\left(\frac{S}{2}-x\right), \mathrm{m} / \mathrm{s}^{2}
$$

For $x=0$ and $x=S$ the maximum accelerations are obtained $a_{\max }=\frac{S \omega^{2}}{2}$.

The maximum inertia force of the $M$ mass knife is

$$
F_{i \max }=\frac{M S \omega^{2}}{2}
$$

\section{Results}

When making the equipment, a number of theoretical considerations were addressed on the factors that influence the hemp harvesting process: the cutting devices of the harvesting machines; the kinematics of the cutting knife, the cutting speed; the critical angle of plant catching, the cutting angles of the cutting blades, the forces acting on the knife, in a study on which the machine design was based.

Based on the theoretical elements, the Equipment for harvesting green hemp stalks, ERCV Figure 4, was developed, which is composed of the following subassemblies:

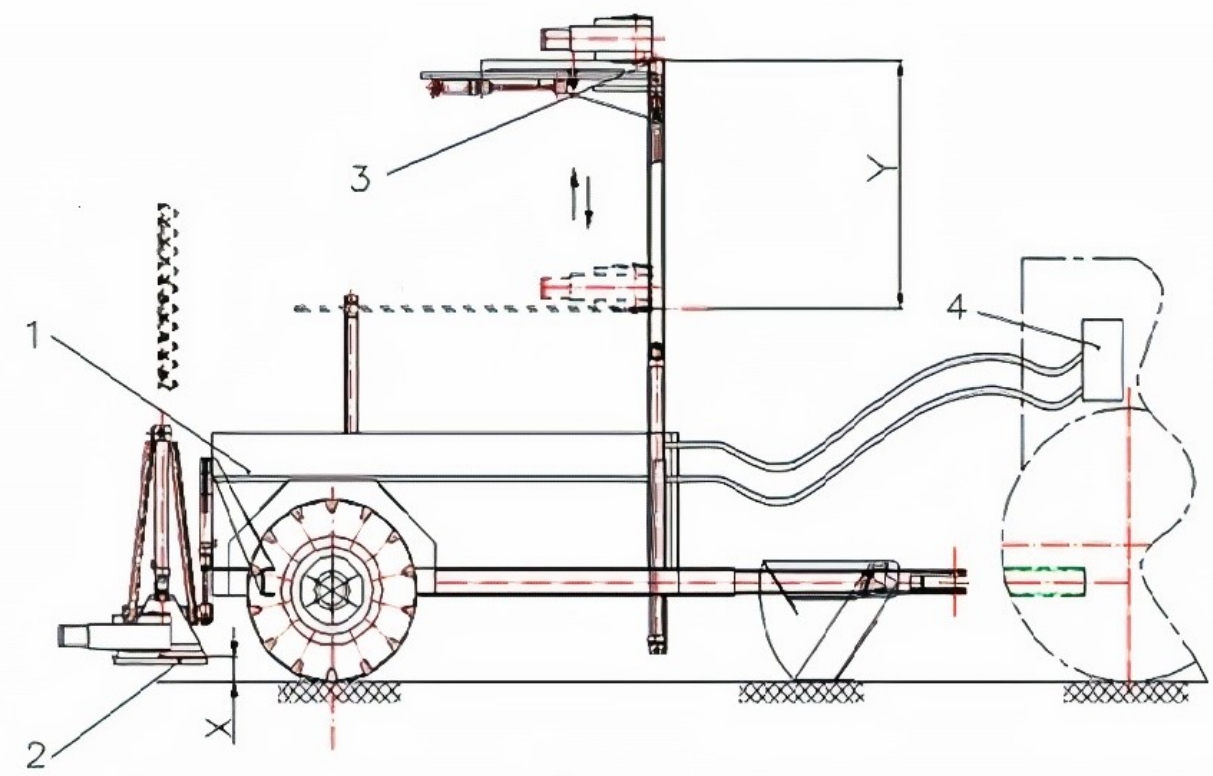

Fig. 4. Equipment for harvesting green hemp stalks ERCV: 1 - Platform; 2 - Lower cutting machine; 3 - Higher cutting machine; 4 - Hydraulic installation

Before entering the hemp crop, the tractor is started and the two cutting devices are actuated by means of the hydraulic system. They are brought from the transport position to 
the working position. The first cutting machine (rear platform) is set for cutting the stalks at a height of $100 \mathrm{~mm}$ from the ground.

Depending on the height of the hemp plant, respectively the inflorescence of the plant, the second cutting device (front platform), is adjusted vertically by means of a vertical cylinder. When the equipment operates, the cut plants remain on the ground, with the inflorescence mixed with the stalks.

In the transport position, the cutting machine is folded down and raised vertically by actuating with the help of two hydraulic cylinders working at the ends of the stroke. The second device is mounted horizontally offset to the first, in the front of the platform and performs the cutting of hemp plants' inflorescences. Because the height of the hemp varieties' inflorescences varies, this device has the possibility of adjusting the cutting height in a wide range of values with the help of a vertical cylinder. The second hydraulic cylinder works at the ends of the stroke in the working or transport position of the equipment. During transport this unit can be folded 90 degrees backwards on a support, with the help of the hydraulic system. Both knives are double-cut devices.

Each cutting device is actuated by a hydraulic motor through a distributor, operated from the hydraulic system of the equipment.

General technical characteristics of ERCV-0

- Machine type

trailed

- Energy source

65 HP tractor

- Type of cutting machine

Double-knife

- No. of cutting devices

2

- Cutting height:

- rear cutting machine, $\mathrm{mm}$

100

- front cutting machine, $\mathrm{mm}$

1500-2500

\section{Conclusions}

Knowing the characteristics of the hemp stalks that were subject of the study allowed establishing the constructive solutions that would respond to the demands of hemp growers;

Theoretical considerations on the factors that influence the process of hemp harvesting: the cutting devices of the harvesting machines; the kinematics of the cutting knife, the cutting speed; the critical angle of plant catching, the cutting angles of the cutting blades, the forces acting on the knife, approached allowed establishing the technical and functional characteristics of the equipment for harvesting green hemp stalks.

Through the constructive solutions adopted, the aim was to create a piece of equipment that would provide the following technological requirements in the case of hemp harvesting for fibre: the average cutting height from the ground should not exceed $100 \mathrm{~mm}$, stalk losses should be less than $5 \%$, and the percentage of broken stalks should not be more than $6 \%$, on the other hand and the realization at a lower cost price than the external ones.

This research work was supported by:

A grant of the Romanian Research and Innovation Ministry, PN 191001 03-Substantion of the technology for harvesting and primary processing green hemp stalks, contract no. 5N/07.02.2019.

A grant of the Romanian Education and Research Ministry, through Programme 1 - Development of the national research-development system, sub-programme 1.2 - Institutional performance Projects for financing excellence in RDI, contract no. 16PFE. 


\section{References}

1. Carus M., Karst S., Kauffmann A. The European Hemp Industry: Cultivation, Processing and Applications for Fibres, Shives and Seeds. Cologne: EIHA, pp. 1-9, (2013).

2. Amaducci S., Zatta A., Pelatti F., Venturi G. Influence of agronomic factors on yield and quality of hemp (Cannabis sativa L.) fibre and implication for an innovative production system (Field Crops Res. 107, pp 161-169, 2008).

3. Struik P. C., Yin X., Thouminot C., Bjelková M., Stramkale V., et al. Comparing hemp (Cannabis sativa L.) cultivars for dual-purpose production under contrasting environments (Ind. Crops Prod. 87, pp 33-44, 2016).

4. Păun A., Bogdanof G., Ganea-Christu I., Ciupercă R., Matache M. Patent application no. A-00426/04.07.2019 Equipment for harvesting green hemp / Echipament de recoltat cânepă verde, OSIM, Romania, (2019).

5. Păun, A., Stroescu Ghe., Zaica Al., Technological study on the process of green hemp harvesting, project PN 191001 03-Substantion of the technology for harvesting and primary processing green hemp stalks, INMA, Bucharest, (2019).

6. Luigi Pari a, Przemyslaw Baraniecki b, Ryszard Kaniewski b, Antonio Scarfonea, Harvesting strategies of bast fiber crops in Europe and in China, (Industrial Crops and Products, Volume 68, June 2015, pp 90-96).

7. Bogdanof G., Păun A., Brăcăcescu C., Stroescu Ghe. Considerations on the hydraulic installation of the equipment for harvesting green hemp stalks, ISSN 1454 - 8003, Proceedings of 2019 International Conference on Hydraulics and Pneumatics HERVEX , November 13-15, Băile Govora, Romania, (2019).

8. Huang Jicheng, Shen Cheng, Li Xianwang, Tian Kunpeng, Chen Qiaomin, Zhang Bin, Design and tests of hemp harvester, (International Agricultural Engineering Journal Vol. 26, No. 2, June, (2017). 